\title{
Presbyvestibulopathy: Diagnostic criteria Consensus document of the classification committee of the Bárány Society
}

\author{
Yuri Agrawal ${ }^{\mathrm{a}, *}$, Raymond Van de Berg ${ }^{\mathrm{b}}$, Floris Wuyts ${ }^{\mathrm{c}}$, Leif Walther ${ }^{\mathrm{d}}$, Mans Magnusson ${ }^{\mathrm{e}}$, \\ Esther $\mathrm{Oh}^{\mathrm{f}}$, Margaret Sharpe ${ }^{\mathrm{g}}$ and Michael Strupp ${ }^{\mathrm{h}}$ \\ ${ }^{a}$ Department of Otolaryngology-Head and Neck Surgery, Johns Hopkins University, Baltimore, USA \\ ${ }^{\mathrm{b}}$ Department of Otolaryngology-Head and Neck Surgery, Maastricht University Medical Centre, Maastricht, \\ The Netherlands \\ ${ }^{\mathrm{c}}$ Lab for Equilibrium Investigations and Aerospace, University of Antwerp, Antwerp, Belgium \\ ${ }^{\mathrm{d}}$ Department of Otorhinolaryngology-Head and Neck Surgery, University Medicine Mannheim, \\ University of Heidelberg, Germany \\ ${ }^{\mathrm{e}}$ Department of Otorhinolaryngology, Lund University, Lund, Sweden \\ ${ }^{\mathrm{f}}$ Department of Geriatric Medicine and Gerontology, Johns Hopkins University, Baltimore, USA \\ ${ }^{\mathrm{g}}$ Dizziness and Balance Disorders Centre, Adelaide, Australia \\ ${ }^{\mathrm{h}}$ Department of Neurology and German Center for Vertigo, Ludwig Maximilians University, Munich Germany
}

\begin{abstract}
This paper describes the diagnostic criteria for presbyvestibulopathy (PVP) by the Classification Committee of the Bárány Society. PVP is defined as a chronic vestibular syndrome characterized by unsteadiness, gait disturbance, and/or recurrent falls in the presence of mild bilateral vestibular deficits, with findings on laboratory tests that are between normal values and thresholds established for bilateral vestibulopathy.

The diagnosis of PVP is based on the patient history, bedside examination and laboratory evaluation. The diagnosis of PVP requires bilaterally reduced function of the vestibulo-ocular reflex (VOR). This can be diagnosed for the high frequency range of the VOR with the video-HIT (vHIT); for the middle frequency range with rotary chair testing; and for the low frequency range with caloric testing.

For the diagnosis of PVP, the horizontal angular VOR gain on both sides should be $<0.8$ and $>0.6$, and/or the sum of the maximal peak velocities of the slow phase caloric-induced nystagmus for stimulation with warm and cold water on each side should be $<25 \%$ and $>6 \%$, and/or the horizontal angular VOR gain should be $>0.1$ and $<0.3$ upon sinusoidal stimulation on a rotatory chair.

PVP typically occurs along with other age-related deficits of vision, proprioception, and/or cortical, cerebellar and extrapyramidal function which also contribute and might even be required for the manifestation of the symptoms of unsteadiness, gait disturbance, and falls. These criteria simply consider the presence of these symptoms, along with documented impairment of vestibular function, in older adults.
\end{abstract}

\footnotetext{
${ }^{*}$ Corresponding author: Yuri Agrawal, MD MPH FACS, Department of Otolaryngology-Head and Neck Surgery, Johns Hopkins University School of Medicine, 601 North Caroline Street, 6th Floor Outpatient Center, Baltimore, MD 21287. USA. Tel.: +01 410502 3107; Fax: +01 410955 0035; E-mail: yagrawa1@jhmi.edu.
}

\section{Introduction}

The aging of the global population is a major demographic shift occurring at the present time. As such, the public health impact of conditions that disproportionately affect older adults has been being increasingly recognized. There is growing awareness 
about the consequences of aging of the human sensory systems on individual and public health [1]. Age-related vision loss (due to age-related loss of lens accommodation or "presbyopia," cataracts, or macular degeneration) has been associated with increased risks of disability, falls, and institutionalization [2-4]. Age-related hearing loss (or presbycusis) has been associated with increased risks of dementia, depression, and mortality [5-7]. Studies suggest that age-related deterioration in peripheral sensory structures and the resulting sensory impairments occur commonly in older adults: $15 \%$ of individuals age $\geq 70$ years have symptomatic vision impairment and $26 \%$ of individuals age $\geq 70$ years have symptomatic hearing impairment [8].

A number of studies also demonstrate that aging substantially affects the vestibular sensory system. Moreover, population-based studies suggest that vestibular impairment is highly prevalent in older adults, with nearly $50 \%$ of individuals over age 60 demonstrating some form of vestibular physiologic loss [9, 10]. Indeed, dizziness and imbalance are among the most common symptoms in communitydwelling older adults [11-14]. Age-related decline in vestibular function is known to have important consequences in older adults, notably postural imbalance, gait impairment, and falls [15-20]. Additionally, reduced vestibular function in older adults has been shown to predict reduced ability to carry out activities of daily living and a substantial reduction in quality of life [21].

Given growing evidence of the prevalence and impact of age-related vestibular loss, we propose codifying the definition of presbyvestibulopathy (PVP), as has been done for the aging of other sensory systems (e.g. presbyopia, presbycusis). The PVP diagnosis is intended to encompass mild or incomplete vestibular losses attributable to the normative aging process, consistent with other age-related sensory losses such as presbycusis or presbyopia which are similarly incomplete losses (i.e. in contrast to deafness, or blindness, respectively). Developing formal diagnostic criteria for PVP will be useful in both the clinical and research settings. In the clinical context, providing older adults with symptomatic vestibular impairment with a potential diagnosis of PVP may result in the increased prescription of vestibular treatment, in particular early and continuous vestibular rehabilitation. Additionally, developing uniform diagnostic criteria will allow for standardization of research studies and greater ability to compare and pool subjects across studies.
Other terms which have been used to describe the phenomenon of age-related vestibular loss include presbystasis, presbyequilibrium, presbylibrium, presbyotoconia, and presbyvertigo.

\section{Methods}

Development of the PVP diagnostic criteria followed the process established by the Classification Committee of the Bárány Society (CCBS), which was charged with establishing an International Classification of Vestibular Disorders (ICVD) [22]. The diagnosis was proposed to the CCBS in 2017. The CCBS, which is a multi-national, multi-disciplinary group of experts, evaluated the relevant literature and rationale for establishing a PVP definition and established a subcommittee to develop formal diagnostic criteria. Over the next year, a subcommittee representing three continents and diverse specialties (Otolaryngology, Neurology, Physical Therapy and Geriatrics) was convened. A thorough review of the literature was conducted, and draft criteria were developed.

The criteria are fully supported by notes, comments and written discussion according to the template established by the CCBS for the ICVD. The draft criteria were reviewed by the CCBS and were also made available for a period of public comment. Following an iterative process of refinement and further review, the criteria were finalized for publication. The authors were particularly committed to developing a document on PVP that could be practically applied in diverse clinical settings around the world, advance patient care and outcomes, and establish a clear research agenda.

\section{Diagnostic criteria for presbyvestibulopathy (PVP)}

\section{Each of the criteria A through D have to be fulfilled}

A. Chronic vestibular syndrome (at least 3 months duration) with at least 2 of the following symptoms: ${ }^{1}$

\footnotetext{
${ }^{1}$ Age-related conditions often involve simultaneous impairment of multiple organ systems, as well as a reduced ability to compensate for physiologic losses [23, 24]. In this context, PVP likely occurs simultaneously with other sensory and functional losses (specifically visual, proprioceptive, lower extremity strength, cortical, extrapyramidal, cerebellar), [25-32] which
} 
1. Postural imbalance or unsteadiness

2. Gait disturbance

3. Chronic dizziness

4. Recurrent falls

B. Mild ${ }^{2}$ bilateral peripheral vestibular hypofunction documented by at least 1 of the following:

1. VOR gain measured by video-HIT between 0.6 and $0.8^{3}$ bilaterally

2. VOR gain between 0.1 and 0.3 upon sinusoidal stimulation on a rotatory chair $\left(0.1 \mathrm{~Hz}, \mathrm{Vmax}=50-60^{\circ} / \mathrm{sec}\right)^{4}$

3. Reduced caloric response (sum of bithermal maximum peak SPV on each side between 6 and $\left.25^{\circ} / \mathrm{sec}\right)^{5,6}$

collectively result in the age-related symptoms of postural imbalance, gait disturbance, or recurrent falls [33]. As such, we cannot definitively know the extent to which PVP is contributing to the symptoms. These criteria simply consider the presence of these symptoms, along with documented vestibular physiologic loss, in older adults. Postural imbalance or unsteadiness can refer to static (e.g. standing still) and dynamic imbalance (e.g. standing upright and throwing a ball). Gait disturbance can include slowness and/or unsteadiness of gait. Chronic dizziness refers to symptoms that are systematically present on head movement or walking or when upright. Recurrent falls refers to more than one fall in one year.

${ }^{2}$ We define "mild" vestibular loss as in between normal vestibular function and the level of vestibular loss associated with bilateral vestibulopathy (BVP). We note that individuals should undergo vestibular testing without having recently taken a sedative medication (e.g. benzodiazepine), which can be commonly prescribed in older individuals.

${ }^{3}$ Video-HIT: Quantification of the angular vestibulo-ocular reflex (aVOR) gain is possible with the video head impulse test (vHIT) $[34,35]$. The ICVD definition of BVP defined a VOR gain $<0.6$ bilaterally as one of the diagnostic criteria for BVP [27]. The lower aVOR gain threshold for PVP, which is a mild vestibulopathy, was therefore set at 0.6 . An upper limit of 0.8 was selected as the upper bound by synthesizing data across several studies. One study evaluated aVOR gain in a normative sample of 62 adults, and observed that the lower limit of normal horizontal aVOR gain (2SD below the mean) was 0.79 at $80 \mathrm{~ms}$ and 0.75 at $60 \mathrm{~ms}$ [36]. However, another study of 243 healthy older adults found that an aVOR gain value of $<0.9$ was associated with a significantly higher prevalence of compensatory saccades, which are considered a marker of VOR deficiency [37]. In order to capture the mild vestibular loss consistent with PVP, we are setting the aVOR gain thresholds to $\geq 0.6$ and $<0.8$.

${ }^{4}$ Rotary chair: Rotary chair testing measures aVOR responses to low to mid-frequency stimulation $(\sim 0.05$ to $0.1 \mathrm{~Hz})$. We again use the BVP cutoff of aVOR gain $\geq 0.1$ as the lower threshold. For the upper threshold, we selected the upper threshold of $<0.3$ given that $0.3-0.35$ are considered the lower range of normal in many laboratories [38].
C. Age $\geq 60$ years $^{7}$
D. Not better accounted for by another disease or disorder $^{8}$

\section{Comments}

\section{Epidemiology}

\subsection{Prevalence}

By $2050,17 \%$ of the world's population will be age 65 and older, equivalent to 1.6 billion individuals [49]. As such, there is a critical need to recognize how vestibular function changes in older individuals and how these changes may manifest clinically and in the population. Vestibular impairment typically manifests as dizziness, imbalance, or vertigo, which are highly prevalent symptoms among older adults although are not specific to vestibular

\footnotetext{
${ }^{5}$ Caloric response: Caloric testing measures low-frequency $(\sim 0.003 \mathrm{~Hz})$ aVOR responses. The bilateral vestibulopathy criteria defined $<6^{\circ} / \mathrm{sec}$ for both warm $\left(44^{\circ} \mathrm{C}\right)$ and cool $\left(30^{\circ} \mathrm{C}\right)$ responses in each ear as a diagnostic threshold [39]. As such, we define $\geq 6 \%$ sec as the lower threshold for caloric responses. We define the upper threshold as just below the normal cutoff defined by numerous laboratories: $<25^{\circ} / \mathrm{sec}$ for both warm and cool responses in each ear [40]. In other words, individuals should have a combined (sum of warm and cool) peak SPV of $\geq 6 \% \mathrm{sec}$ and $<25 \%$ sec in both ears.

${ }^{6}$ We did not include otolith impairment as part of the diagnostic criteria, despite evidence that suggests decline in otolith function associated with age. As discussed below in the section on Laboratory Testing, at this time clinical testing of otolith function has not been operationalized and standardized to the same extent as VOR testing through caloric, rotary chair and vHIT testing. However, the inclusion of otolith impairment will be revisited in subsequent revisions of these diagnostic criteria.

${ }^{7} 60$ years was chosen as the age cutoff for PVP based on the United Nations cutoff for older adults [41]. Moreover, histologic studies have documented degradation of vestibular end-organ structure at age 60. A marked decline in vestibular hair cell populations was observed throughout the canal and otolith sensory epithelia beginning at age 50 [42]. Degeneration of saccular and utricular otoconia has also been documented beginning at age 50 [43]. Significant reductions in vestibular nerve fibers have been observed starting in the 5th decade of life, [44] while neuronal loss within the vestibular nuclei begins at $\sim$ age 40 and ganglion cell counts start declining roughly at age $60[45,46]$.

${ }^{8}$ It should be noted that some individuals may have multiple diagnoses, such as BPPV (which increases in prevalence with age), or a neurological disease (e.g. Parkinson's disease), as well as PVP $[47,48]$. The critical point is that the other diagnosis may present with additional symptoms (e.g. brief, positional vertigo for BPPV, bradykinesia and rigidity for Parkinson's disease), but the other diagnosis does not fully account for the diagnostic criteria of PVP.
} 
dysfunction. Estimates of the prevalence of dizziness and imbalance in the older population depend largely on the definitions of dizziness and imbalance used, and on the populations surveyed. Several large population-based studies reported a $20-30 \%$ prevalence of dizziness and imbalance in the elderly population (age $\geq 65$ years) [12-14]. The prevalence of dizziness and imbalance was found to rise steeply with age, with levels over $50 \%$ in the communitydwelling population over age 80 [11]. A study in institutionalized nursing home residents observed a prevalence of dizziness and vertigo of $68 \%$ [19]. Among patients age $\geq 65$ presenting to a geriatric primary care clinic, $24 \%$ reported dizziness and $17 \%$ identified dizziness as their major presenting complaint [50].

A landmark series of studies based in Germany estimated the population prevalence and incidence more specifically of vestibular vertigo, i.e. vertigo resulting from vestibular impairment. Among community-dwelling individuals age $\geq 18$ years, the lifetime prevalence, 1-year prevalence and incidence of vestibular vertigo were observed to be $7.8 \%, 4.9 \%$ and $1.5 \%$ respectively [51]. The 1 -year prevalence of vestibular vertigo increased with age to $7.2 \%$ in 60-69 year-olds and $8.8 \%$ in individuals over age 80 . Another study estimated the prevalence of vestibular impairment in the US population using the objective modified Romberg test. Thirty-five percent of US adults age 40 years and older had evidence of vestibular dysfunction based on this postural metric [52]. The frequency of balance dysfunction increased significantly with age, such that $85 \%$ of individuals age 80 and above had evidence of balance dysfunction. These estimates are considerably higher than the prevalence of vestibular vertigo reported above from the German population, possibly because the modified Romberg test captures subclinical vestibular impairment, and may reflect more broadly the inability to use vestibular information.

\subsection{Impact}

The impacts of vestibular impairment in older adults have also been characterized in numerous studies. Vestibular impairment in older adults has been associated with reduced gait speed, and reduced postural control $[15,53]$. Emerging evidence also suggests that reduced vestibular function in older individuals is associated with poorer cognition, notably spatial cognition $[17,54,55]$. Further, agerelated vestibular loss has been shown to increase the risk of falls, which is a common and devastating outcome in older adults [56, 57]. Several studies observed an association between vestibular impairment and fall-related hip and wrist fracture risk [58-60]. One recent study estimated that about 50,000 excess falls per year in older adults could be attributable to vestibular loss [61].

Additionally, dizziness and vestibular impairment have been associated with difficulty carrying out basic and instrumental activities of daily living, such as getting in and out of bed, driving, shopping, and even managing money $[11,21]$. With respect to health care utilization and economic outcomes, vestibular vertigo was associated with increased likelihood of a medical consultation, taking sick leave, and avoidance of leaving the house [62]. Finally, dizziness and vestibular impairment have been associated with significantly poorer quality of life, in both the physical and mental domains $[62,63]$. One population-based study in Sweden found that dizziness was one of the most influential symptoms affecting general quality of life in older individuals [64].

Taken together, these studies demonstrate the broad prevalence and substantial impacts of vestibular loss in older adults and underscore the public health relevance of this condition. Moreover, we note that although dizziness, imbalance and vestibular impairment are prevalent in older adults they are not universal. Not all individuals over age 90 had dizziness, [11] and healthy older adults have been shown to be able to maintain a normal VOR gain even over age 80 [65]. As such, dizziness, imbalance and vestibular impairment in the older population may be considered "age-concomitant" rather than "agedependent" conditions [11]. As a corollary, these conditions should be viewed as potentially modifiable, and efforts should be made to treat them. Indeed, a strong body of evidence supports the benefit of vestibular rehabilitation in older patients with vestibular physiologic loss, [66] as well as in older adults with dizziness more broadly who have not been specifically screened for vestibular impairment [67].

\section{Pathophysiology}

Numerous lines of evidence demonstrate a decline in vestibular sensory function with aging. These age-related declines in structural and physiological vestibular function are thought to be due to endogenous factors (e.g. genetic) and the cumulative exposure to vestibulotoxic agents including 
infection, inflammation, vasculopathy, medications, and trauma. Histopathologic studies have long documented age-related declines in vestibular sensory epithelia throughout the vestibular end-organ (e.g. declining hair cell counts in the three semicircular canals and the utricle and saccule and morphological changes in the otoconia), as well as declines in vestibular ganglion cell, afferent, and vestibular nucleus cell populations [68-70].

Physiologic studies that have assessed vestibular responses to rotational, translational, acoustic and vibratory stimulation have also observed declining response amplitudes and increasing response latencies associated with age [61, 71-77]. The earliest studies of vestibular physiologic function and aging examined responses to sinusoidal rotation in cross-sectional samples across the age range. The studies reported decreased VOR gain to sinusoidal rotation with age, [71] and the authors concluded that "aging entails a progressive bilateral peripheral vestibular loss" [73]. Decline in VOR processing mechanisms such as velocity storage were also noted with increased age [78]. Subsequent longitudinal studies in healthy older individuals observed decreasing VOR responses to sinusoidal rotation within individuals over a 5-year period [79, 80]. More recent studies have used newer, portable vestibular testing methods such as the video-head impulse test (vHIT) to measure the VOR in large population-based samples of older adults, and reductions in angular VOR gain associated with age have been reported [36, $81,82]$. Moreover, declines in otolith function have also been reported, as measured by a number of tests of otolith function including vestibular-evoked myogenic potentials (VEMPs), vestibular linear perceptual threshold testing, and otolith-ocular reflexes $[61,74,83]$.

\section{Differential diagnosis}

The differential diagnosis of PVP is provided in Table 1. Although many of the diagnoses can be distinguished from PVP by the absence of bilateral vestibular testing deficits, we note that most of these conditions (with the exception of unilateral vestibulopathy and BVP) can co-occur with PVP. Indeed, a hallmark of aging is the frequent co-existence of multiple deficits that all contribute to the observed phenotype.

Of these diagnoses, benign paroxysmal positional vertigo (BPPV) is particularly common in older adults and bears special mention. Increased prevalence of BPPV in the elderly may reflect age-related degeneration of the otoconial membrane, leading to abnormal seeding of otoconia in the endolymph [84]. A population-based study observed a prevalence of BPPV of $3.4 \%$ in individuals over age 60 , and a cumulative lifetime incidence of almost $10 \%$ by age 80 [47]. BPPV accounted for up to $39 \%$ of cases of vertigo in older patients presenting to neuro-otology clinics [85]. However, older patients do not always experience the classic presentation of BPPV, i.e., short episodes of rotatory vertigo associated with changes in head position. A study of 100 older patients presenting to general geriatric practices for chronic medical conditions found that $9 \%$ had unrecognized BPPV [48]. As such, careful clinical assessment for BPPV is warranted in older adults presenting with dizziness as it is a common and treatable condition.

\section{Current knowledge gaps and future directions}

\subsection{Symptoms}

The PVP classification committee chose to include symptoms in the diagnostic criteria that are most commonly associated with vestibular impairment, including postural imbalance, gait abnormality, dizziness, and recurrent falls. These are all patientreported symptoms elicited during a typical clinic visit. However, there is no quantification of the severity of the symptoms. Possible objective measures of postural imbalance include the Romberg test, tandem stance test, and platform posturography. Objective measures of gait abnormality include gait speed, tandem walking, and indices such as the Dynamic Gait Index and the Functional Gait Assessment [86]. Dizziness related handicap can be further specified using patient-reported instruments such as the Dizziness Handicap Inventory [87]. Falls can be quantified based on number of falls over a specified time period, or further characterized based on patientreported instruments such as the Falls Efficacy Scale [88]. At present, the PVP Classification Committee decided that there is insufficient standardization of these measures, as well as a lack of broadly recognized thresholds of normal vs. abnormal values in older adults, to justify the inclusion of specific symptom metrics. Future revisions of these diagnostic criteria should reassess which more detailed 
Table 1

Differential diagnosis of PVP

\begin{tabular}{|c|c|}
\hline Differential diagnosis of PVP & Distinction from PVP \\
\hline \multicolumn{2}{|l|}{ Other vestibular } \\
\hline Benign paroxysmal positional vertigo & Positive Dix-Hallpike/diagnostic Semont maneuver or supine roll test \\
\hline Persisting unilateral vestibulopathy & PVP is bilateral \\
\hline Bilateral vestibulopathy & Vestibular deficits in PVP not as severe as BVP \\
\hline Functional dizziness (e.g. PPPD, visual dizziness) & Absence of bilateral vestibular testing deficits \\
\hline \multicolumn{2}{|l|}{ Other sensorimotor } \\
\hline Orthostatic dizziness & Absence of bilateral vestibular testing deficits \\
\hline Low vision & Absence of bilateral vestibular testing deficits \\
\hline Proprioceptive impairment & Absence of bilateral vestibular testing deficits \\
\hline \multicolumn{2}{|l|}{ Central nervous system } \\
\hline Cerebellar ataxia without bilateral vestibulopathy & Absence of bilateral vestibular testing deficits \\
\hline Downbeat nystagmus syndrome & $\begin{array}{l}\text { Presence of downbeat nystagmus, with or without peripheral vestibular } \\
\text { deficits }\end{array}$ \\
\hline Extrapyramidal disorders & $\begin{array}{l}\text { Extrapyramidal symptoms (e.g. rigidity, bradykinesia), with or without } \\
\text { peripheral vestibular deficits }\end{array}$ \\
\hline Normal pressure hydrocephalus (NPH) & $\begin{array}{l}\text { Symptoms of NPH, hydrocephalus, positive tap test with or without } \\
\text { peripheral vestibular deficits }\end{array}$ \\
\hline Vestibular suppressant medications & Absence of bilateral vestibular testing deficits \\
\hline \multicolumn{2}{|l|}{ Systemic } \\
\hline Intoxications & Absence of bilateral vestibular testing deficits \\
\hline
\end{tabular}

measures could be added to the PVP diagnostic criteria.

Moreover, emerging literature is demonstrating the impact of vestibular impairment in older adults on cognitive function, notably spatial cognition which encompasses spatial memory, spatial navigation and spatial orientation [17, 89]. However, the PVP Classification Committee determined that cognitive function is influenced by a broad network of central nervous system and peripheral factors, and that further evidence is still needed to demonstrate a direct, link between vestibular impairment and cognition. Future revisions of these diagnostic criteria should reassess whether cognitive impairment should be additionally included to support the diagnosis of PVP, and what measure(s) should be used to ascertain cognitive function.

\subsection{Laboratory testing}

As noted previously, studies have shown that otolith function declines with age, when considering both otolith structure (e.g. utricular and saccular hair cell, otoconial integrity) and function (e.g. as measured by VEMP,and perceptual threshold testing). Moreover, evidence suggests that otolith impairment is associated with postural abnormalities as well as impairment in spatial cognition [17, 74]. However, clinical testing of otolith function has not been operationalized and standardized to the same extent as VOR testing through caloric, rotary chair and vHIT testing. VEMP responses, which are the most widely-used measure of otolith function, can be difficult to elicit reliably. Additionally, VEMP responses are frequently absent in older adults, and the significance of this is not fully understood [90]. A recent position paper from the American Academy of Neurology stated that the clinical utility of VEMPs at present is best characterized for the diagnosis of superior canal dehiscence syndrome and other inner ear third window conditions [24]. As such, the PVP Classification Committee decided to exclude otolith impairment from the PVP diagnostic criteria. Future revisions of these criteria should reassess the state of otolith function testing and re-evaluate the inclusion of otolith impairment in these diagnostic criteria.

For the PVP diagnostic criteria, we selected laboratory values for vHIT, rotary chair, and caloric testing that are above those of BVP and below the lower values of typical normal ranges. Our goal was to identify a level of mild vestibular impairment that is analogous to presbycusis or presbyopia, which are distinct from profound sensory losses (i.e. deafness or blindness). However, it is unknown for each of these vestibular tests whether there are specific thresholds of responses that are associated with the onset of clinical symptoms. Future revisions of these criteria should consider whether the cutoffs for mild vestibular impairment in older adults should be refined. 
A further consideration for future revisions of these diagnostic criteria is the delineation of subtypes of PVP, e.g. with respect to semicircular canal vs. otolith impairment, low-frequency vs. highfrequency vestibular impairment, and peripheral vs. central vestibular impairment. For example, presbycusis is known to be a high frequency hearing loss, and analogously within the vestibular sensory system the high-frequency responsive Type 1 hair cells are known to disproportionately degrade with age relative to the Type 2 low-frequency responsive hair cells [69]. Additionally, histopathological studies suggest that semicircular canal hair cells degenerate to a greater extent than otolith hair cells with age, a finding that has been corroborated in clinical studies $[9,68]$. Future revisions of these diagnostic criteria should consider whether PVP can be more precisely defined with respect to the types of vestibular physiologic impairment that occur with aging.

\subsection{The aging phenotype}

As noted, aging is characterized by concomitant declines across multiple physiologic systems over time. As such, vestibular hypofunction can be expected to occur along with other sensorimotor, CNS, and systemic declines. Although these diagnostic criteria focus on reductions on vestibular physiologic function, it is likely that the clinical impact of PVP is modulated by the functional state of other physiologic systems. For example, older adults may have reduced ability to compensate for the same level of peripheral vestibular hypofunction, based on their existing sensorimotor function, their ability to reweight sensory inputs, and their level of CNS plasticity. Future revisions of these diagnostic criteria may more explicitly consider PVP in the context of other physiologic systems and their joint effects on functional impairment in older adults.

\section{References}

[1] Y. Agrawal, J.P. Carey, C. Della Santina, M.C. Schubert and L.B. Minor, Disorders of balance and vestibular function in US adults: Data from the National Health and Nutrition Examination Survey, 2001-2004, Arch Intern Med 169(10) (2009), 938-944.

[2] Y. Agrawal, M. Davalos-Bichara, M.G. Zuniga and J.P. Carey, Head impulse test abnormalities and influence on gait speed and falls in older individuals, Otology \& Neurotology 34(9) (2013), 1729-1735.
[3] Y. Agrawal, K.G. Pineault and Y.R. Semenov, Healthrelated quality of life and economic burden of vestibular loss in older adults, Laryngoscope Investigative Otolaryngology. 2017.

[4] Y. Agrawal, M.G. Zuniga, M. Davalos-Bichara, M.C. Schubert, J.D. Walston, J. Hughes, et al. 3376350; Decline in semicircular canal and otolith function with age, $\mathrm{Otol} \mathrm{Neu-}$ rotol 33(5) (2012), 832-839.

[5] E.R. Anson, R.T. Bigelow, J.P. Carey, Q.L. Xue, S. Studenski, M.C. Schubert, et al., 4919329; VOR Gain Is Related to Compensatory Saccades in Healthy Older Adults, Front Aging Neurosci 8 (2016), 150.

[6] R.W. Baloh, K.M. Jacobson and T.M. Socotch, The effect of aging on visual-vestibuloocular responses, Experimental Brain Research 95(3) (1993), 509-516.

[7] R.W. Baloh, J. Enrietto, K.M. Jacobson and A. Lin, Agerelated changes in vestibular function: A longitudinal study, Ann N Y Acad Sci 942 (2001), 210-219.

[8] R.W. Baloh, S.H. Ying and K.M. Jacobson, A longitudinal study of gait and balance dysfunction in normal older people, Arch Neurol 60(6) (2003), 835-839.

[9] P.B. Baltes and U. Lindenberger, Emergence of a powerful connection between sensory and cognitive functions across the adult life span: A new window to the study of cognitive aging? Psychol Aging 12(1) (1997), 12.

[10] K. Bartl, N. Lehnen, S. Kohlbecher and E. Schneider, Head impulse testing using video-oculography, Ann N Y Acad Sci 1164 (2009), 331-333.

[11] B. Bergström Morphology of the vestibular nerve: II. The number of myelinated vestibular nerve fibers in man at various ages, Acta Otolaryngol 76(1-6) (1973), 173-179.

[12] R.T. Bigelow and Y. Agrawal, Vestibular involvement in cognition: Visuospatial ability, attention, executive function, and memory, Journal of Vestibular Research 25(2) (2015), 73-89.

[13] R.T. Bigelow, Y.R. Semenov, S. du Lac, H.J. Hoffman and Y. Agrawal, Vestibular vertigo and comorbid cognitive and psychiatric impairment: The 2008 National Health Interview Survey, J Neurol Neurosurg Psychiatr (2015), 310319.

[14] R.T. Bigelow, Y.R. Semenov, C. Trevino, L. Ferrucci, S.M. Resnick, E.M. Simonsick, et al., Association between visuospatial ability and vestibular function in the Baltimore Longitudinal Study of Aging, J Am Geriatr Soc 63(9) (2015), 1837-1844.

[15] A. Bisdorff, M. Von Brevern, T. Lempert and D. NewmanToker, Classification of vestibular symptoms: Towards an international classification of vestibular disorders, Journal of Vestibular Research 19(1) (2009), 1-13.

[16] K. Brantberg, K. Granath and N. Schart, Age-related changes in vestibular evoked myogenic potentials, Audiol Neurootol 12(4) (2007), 247-253.

[17] B.L. Brody, A.C. Gamst, R.A. Williams, A.R. Smith, P.W. Lau, D. Dolnak, et al., Depression, visual acuity, comorbidity, and disability associated with age-related macular degeneration, Ophthalmology 108(10) (2001), 1893-1900.

[18] A. Campos, F.J. Canizares, M.C. Sanchez-Quevedo and P.J. Romero, Otoconial Degeneration in the Aged Utricle and Saccule1. In: Karger Publishers; 1990. pp. 143-153.

[19] F.M. Chan, J. Galatioto, M. Amato and A.H. Kim, Normative data for rotational chair stratified by age, Laryngoscope 126(2) (2016), 460-463.

[20] R.G. Cumming, R. Ivers, L. Clemson, J. Cullen, M.F. Hayes, M. Tanzer, et al., Improving vision to prevent falls in frail older people: A randomized trial, J Am Geriatr Soc 55(2) (2007), 175-181. 
[21] C.F. Dillon, Q. Gu, H.J. Hoffman and C. Ko, Vision, hearing, balance, and sensory impairment in Americans aged 70 years and over: United States, 1999-2006, NCHS data Brief (31) (2010), 1-8.

[22] A. Ekwall, A. Lindberg and M. Magnusson, Dizzy - why not take a walk? Low level physical activity improves quality of life among elderly with dizziness, Gerontology $\mathbf{5 5}(6)$ (2009), 652-659.

[23] E. Ekvall Hansson and M. Magnusson, 3724742; Vestibular asymmetry predicts falls among elderly patients with multisensory dizziness, BMC Geriatr 13(1) (2013), 77.

[24] T.D. Fife, J.G. Colebatch, K.A. Kerber, K. Brantberg, M. Strupp, H. Lee, et al., Practice guideline: Cervical and ocular vestibular evoked myogenic potential testing Report of the Guideline Development, Dissemination, and Implementation Subcommittee of the American Academy of Neurology, Neurology (2017), 10.1212/WNL. 0000000000004690.

[25] S.K. Gadkaree, D.Q. Sun, C. Li, F.R. Lin, L. Ferrucci, E.M. Simonsick, et al., Does sensory function decline independently or concomitantly with age? Data from the Baltimore Longitudinal Study of Aging, Journal of Aging Research 2016; 2016.

[26] D.J. Genther, J. Betz, S. Pratt, S.B. Kritchevsky, K.R. Martin, T.B. Harris, et al., Association of hearing impairment and mortality in older adults, Journals of Gerontology Series A: Biomedical Sciences and Medical Sciences 70(1) (2014), $85-90$.

[27] N.S. Gittings and J.L. Fozard, Age related changes in visual acuity, Exp Gerontol 21(4-5) (1986), 423-433.

[28] B. Gopinath, C.M. McMahon, E. Rochtchina and P. Mitchell, Dizziness and vertigo in an older population: The Blue Mountains prospective cross-sectional study, Clinical Otolaryngology 34(6) (2009), 552-556.

[29] A. Grimby and U. Rosenhall, Health-related quality of life and dizziness in old age, Gerontology 41(5) (1995), 286-298.

[30] J.M. Guralnik, L. Ferrucci, E.M. Simonsick, M.E. Salive and R.B. Wallace, Lower-extremity function in persons over the age of 70 years as a predictor of subsequent disability, $N$ Engl J Med 332(9) (1995), 556-561.

[31] A. Harun, Y.R. Semenov and Y. Agrawal, Vestibular function and activities of daily living: Analysis of the 1999 to 2004 National Health and Nutrition Examination Surveys, Gerontology and Geriatric Medicine 1 (2015), 2333721415607124.

[32] R.H. Harwood, Visual problems and falls, Age Ageing 30(Suppl 4) (2001), 13-18.

[33] W. He, D. Goodkind and P. Kowal, An aging world: 2015. International population reports, Last modified March (2016), 31.

[34] G.E. Hicks, M. Shardell, D.E. Alley, R.R. Miller, S. Bandinelli, J. Guralnik, et al., 3260485; Absolute strength and loss of strength as predictors of mobility decline in older adults: The InCHIANTI study, J Gerontol A Biol Sci Med Sci 67(1) (2012), 66-73.

[35] G. Ishiyama, Imbalance and vertigo: The aging human vestibular periphery, Semin Neurol 29(5) (2009), 491-499.

[36] G.P. Jacobson, D.L. McCaslin, S.L. Grantham and E.G. Piker, Significant vestibular system impairment is common in a cohort of elderly patients referred for assessment of falls risk, J Am Acad Audiol 19(10) (2008), 799-807.

[37] G.P. Jacobson and C.W. Newman, The development of the Dizziness Handicap Inventory, Arch Otolaryngol Head Neck 116(4) (1990), 424-427.
[38] A.C. Kao, A. Nanda, C.S. Williams and M.E. Tinetti, Validation of dizziness as a possible geriatric syndrome, $J \mathrm{Am}$ Geriatr Soc 49(1) (2001), 72-75.

[39] A. Katsarkas, Dizziness in aging: A retrospective study of 1194 cases, Otolaryngol Head Neck Surg 110(3) (1994), 296-301.

[40] E.A. Keshner, Head-trunk coordination in elderly subjects during linear anterior-posterior translations, Exp Brain Res 158(2) (2004), 213-222.

[41] E.K. Kristinsdottir, G.B. Jarnlo and M. Magnusson, Asymmetric vestibular function in the elderly might be a significant contributor to hip fractures, Scand J Rehabil Med 32(2) (2000), 56-60.

[42] E.K. Kristinsdottir, E. Nordell, G.B. Jarnlo, A. Tjader, K.G. Thorngren and M. Magnusson, Observation of vestibular asymmetry in a majority of patients over 50 years with fall-related wrist fractures, Acta Otolaryngol 121(4) (2001), 481-485.

[43] C. Li, A.J. Layman, R. Geary, E. Anson, J.P. Carey, L. Ferrucci, et al., Epidemiology of vestibulo-ocular reflex function: Data from the Baltimore Longitudinal Study of Aging. Otology \& neurotology: Official publication of the American Otological Society, American Neurotology Society and] European Academy of Otology and Neurotology 36(2) (2015), 267.

[44] H.W. Lin and N. Bhattacharyya, Balance disorders in the elderly: Epidemiology and functional impact, Laryngoscope 122(8) (2012), 1858-1861.

[45] F.R. Lin, E.J. Metter, R.J. O’Brien, S.M. Resnick, A.B. Zonderman and L. Ferrucci, Hearing loss and incident Dementia, Arch Neurol 68(2) (2011), 214-220.

[46] M.B. Liston, D.E. Bamiou, F. Martin, A. Hopper, N. Koohi, L. Luxon, et al., Peripheral vestibular dysfunction is prevalent in older adults experiencing multiple non-syncopal falls versus age-matched non-fallers: A pilot study, Age Ageing 43(1) (2014), 38-43.

[47] I. Lopez, V. Honrubia and R.W. Baloh, Aging and the human vestibular nucleus, Journal of Vestibular Research 7(1) (1997), 77-85.

[48] H.G. MacDougall, K.P. Weber, L.A. McGarvie, G.M. Halmagyi and I.S. Curthoys, The video head impulse test: Diagnostic accuracy in peripheral vestibulopathy, Neurology 73(14) (2009), 1134-1141.

[49] L. Maes, I. Dhooge, W. D'haenens, A. Bockstael, H. Keppler, B. Philips, et al., The effect of age on the sinusoidal harmonic acceleration test, pseudorandom rotation test, velocity step test, caloric test, and vestibularevoked myogenic potential test, Ear Hear 31(1) (2010), 84-94.

[50] G.F. Marchetti and S.L. Whitney, Older adults and balance dysfunction, Neurol Clin 23(3) (2005), 785-805.

[51] A.J. Matheson, C.L. Darlington and P.F. Smith, Dizziness in the elderly and age-related degeneration of the vestibular system, New Zealand Journal of Psychology 28(1) (1999), 10.

[52] E. Matiño-Soler, E. Esteller-More, J. Martin-Sanchez, J. Martinez-Sanchez and N. Perez-Fernandez, Normative data on angular vestibulo-ocular responses in the yaw axis measured using the video head impulse test, Otology \& Neurotology 36(3) (2015), 466-471.

[53] L.A. McGarvie, H.G. MacDougall, G.M. Halmagyi, A.M. Burgess, K.P. Weber and I.S. Curthoys, The video head impulse test (vHIT) of semicircular canal function-agedependent normative values of VOR gain in healthy subjects, Frontiers in Neurology (2015), 6. 
[54] D.J. Mener, J. Betz, D.J. Genther, D. Chen and F.R. Lin, Hearing loss and depression in older adults, $J$ Am Geriatr Soc 61(9) (2013), 1627.

[55] S.N. Merchant, L. Velazquez-Villasenor, K. Tsuji, R.J. Glynn, C. Wall, 3 and S.D. Rauch, Temporal bone studies of the human peripheral vestibular system. Normative vestibular hair cell data, Ann Otol Rhinol Laryngol Suppl 181 (2000), 3-13.

[56] B. Mossman, S. Mossman, G. Purdie and E. Schneider, Age dependent normal horizontal VOR gain of head impulse test as measured with video-oculography, Journal of Otolaryngology-Head \& Neck Surgery 44(1) (2015), 29.

[57] H.K. Neuhauser, A. Radtke, M. von Brevern, F. Lezius, M. Feldmann and T. Lempert, Burden of dizziness and vertigo in the community, Arch Intern Med 168(19) (2008), 2118-2124.

[58] H.K. Neuhauser, M. von Brevern, A. Radtke, F. Lezius, M. Feldmann, T. Ziese, et al., Epidemiology of vestibular vertigo: A neurotologic survey of the general population, Neurology 65(6) (2005), 898-904.

[59] E. Nordell, E.K. Kristinsdottir, G.B. Jarnlo, M. Magnusson and K.G. Thorngren, Older patients with distal forearm fracture. A challenge to future fall and fracture prevention, Aging Clin Exp Res 17(2) (2005), 90-95.

[60] J.S. Oghalai, S. Manolidis, J.L. Barth, M.G. Stewart and H.A. Jenkins, Unrecognized benign paroxysmal positional vertigo in elderly patients, Otolaryngol Head Neck Surg 122(5) (2000), 630-634.

[61] G.D. Paige, Senescence of human visual-vestibular interactions. 1. Vestibulo-ocular reflex and adaptive plasticity with aging, J Vestib Res 2(2) (1992), 133-151.

[62] R.J. Peterka, F.O. Black and M.B. Schoenhoff, Age-related changes in human vestibulo-ocular and optokinetic reflexes: Pseudorandom rotation tests, J Vestib Res 1(1) (1990), 61-71.

[63] S.D. Rauch, L. Velazquez-Villasenor, P.S. Dimitri and S.N. Merchant, Decreasing hair cell counts in aging humans, Ann N Y Acad Sci 942 (2001), 220-227.

[64] M.C.B. Rey, T.K. Clark, W. Wang, T. Leeder, Y. Bian and D.M. Merfeld, Vestibular perceptual thresholds increase above the age of 40, Front Neurol (2016), 7.

[65] E. Richter, Quantitative study of human Scarpa's ganglion and vestibular sensory epithelia, Acta Otolaryngol 90(1-6) (1980), 199-208.

[66] S. Robbins, E. Waked and J. McClaran, Proprioception and stability: Foot position awareness as a function of age and footware, Age Ageing 24(1) (1995), 67-72.

[67] R.E. Roditi and B.T. Crane, Directional asymmetries and age effects in human self-motion perception, $J$ Assoc Res Otolaryngol 13(3) (2012), 381-401.

[68] S.M. Rosengren, M.S. Welgampola and J.G. Colebatch, Vestibular evoked myogenic potentials: Past, present and future, Clinical Neurophysiology 121(5) (2010), 636-651.

[69] U. Rosenhall, Degenerative patterns in the aging human vestibular neuro-epithelia, Acta Otolaryngol 76(2) (1973), 208-220.

[70] Y.R. Semenov, R.T. Bigelow, Q. Xue, S. du Lac and Y. Agrawal, Association between vestibular and cognitive function in US adults: Data from the National Health and Nutrition Examination Survey, The Journals of Gerontology Series A: Biological Sciences and Medical Sciences 71(2) (2016), 243-250.

[71] J.M. Serrador, L.A. Lipsitz, G.S. Gopalakrishnan, F.O. Black and S.J. Wood, Loss of otolith function with age is associated with increased postural sway measures, Neurosci Lett 465(1) (2009), 10-15.

[72] H.B. Skinner, R.L. Barrack and S.D. Cook, Age-related decline in proprioception, Clin Orthop 184 (1984), 208-211.

[73] K.N. Stevens, I.A. Lang, J.M. Guralnik and D. Melzer, Epidemiology of balance and dizziness in a national population: Findings from the English Longitudinal Study of Ageing, Age Ageing 37(3) (2008), 300-305.

[74] M. Strupp, J. Kim, T. Murofushi, D. Straumann, J.C. Jen, S.M. Rosengren, et al., Bilateral vestibulopathy: Diagnostic criteria Consensus document of the Classification Committee of the Bárány Society, Journal of Vestibular Research 27(4) (2017), 177-189.

[75] B.K. Swenor, P.Y. Ramulu, J.R. Willis, D. Friedman and F.R. Lin, The prevalence of concurrent hearing and vision impairment in the United States, JAMA Internal Medicine 173(4) (2013), 312-313.

[76] J.R. Tian, I. Shubayev, R.W. Baloh and J.L. Demer, Impairments in the initial horizontal vestibulo-ocular reflex of older humans, Exp Brain Res 137(3-4) (2001), 309-322.

[77] M.E. Tinetti, D. Richman and L. Powell, Falls efficacy as a measure of fear of falling, J Gerontol 45(6) (1990), 239.

[78] E. Tuunainen, D. Poe, P. Jantti, K. Varpa, J. Rasku, E. Toppila, et al., Presbyequilibrium in the oldest old, a combination of vestibular, oculomotor and postural deficits, Aging Clin Exp Res 23(5-6) (2011), 364-371.

[79] United Nations, Department of Economic and, Social Affairs. World Population Ageing 2015. 2015;(ST/ESA/SER.A/390).

[80] A. Van Der Stappen, F.L. Wuyts and P.H. Van De Heyning, Computerized electronystagmography: Normative data revisited, Acta Otolaryngol 120(6) (2000), 724-730.

[81] M. von Brevern, A. Radtke, F. Lezius, M. Feldmann, T. Ziese, T. Lempert, et al., Epidemiology of benign paroxysmal positional vertigo: A population based study, J Neurol Neurosurg Psychiatr 78(7) (2007), 710-715.

[82] L.E. Walther and M. Westhofen, Presbyvertigo-aging of otoconia and vestibular sensory cells, Journal of Vestibular Research 17(2) (2007), 89-92.

[83] D.E. Warren, M.J. Thurtell, J.N. Carroll and M. Wall, Perimetric Evaluation of Saccadic Latency, Saccadic Accuracy, and Visual Threshold for Peripheral Visual Stimuli in Young Compared With Older AdultsPerimetric Evaluation Using Saccadic Eye Movements, Invest Ophthalmol Vis Sci 54(8) (2013), 5778-5787.

[84] M.S. Welgampola and J.G. Colebatch, Vestibulocollic reflexes: Normal values and the effect of age, Clin $\mathrm{Neu}$ rophysiol 112(11) (2001), 1971-1979.

[85] S.L. Whitney, D.M. Wrisley, G.F. Marchetti and J.M. Furman, The effect of age on vestibular rehabilitation outcomes, Laryngoscope 112(10) (2002), 1785-1790.

[86] D.M. Wrisley and N.A. Kumar, Functional gait assessment: Concurrent, discriminative, and predictive validity in community-dwelling older adults, Phys Ther 90(5) (2010), 761-773.

[87] Y.J. Xie, R.T. Bigelow, S.F. Frankenthaler, S. Studenski, S.D. Moffat and Y. Agrawal, Vestibular Loss in Older Adults is Associated with Impaired Spatial Navigation: Data from the Triangle Completion Task. Front Neurol. 2017; Accepted.

[88] Y.J. Xie, E.Y. Liu, E.R. Anson and Y. Agrawal, Age-Related Imbalance Is Associated With Slower Walking Speed: An Analysis From the National Health and Nutrition Examination Survey, J Geriatr Phys Ther 2016 Jun 23. 
[89] L. Yardley, M. Donovan-Hall, H.E. Smith, B.M. Walsh, M. Mullee and A.M. Bronstein, Effectiveness of primary carebased vestibular rehabilitation for chronic dizziness, Ann Intern Med 141(8) (2004), 598-605.
[90] S.H. You, Joint position sense in elderly fallers: A preliminary investigation of the validity and reliability of the SENSERite measure, Arch Phys Med Rehabil 86(2) (2005), 346-352. 